\title{
USINAGEM DE CAMPO DAS PALHETAS DO ULTIMO ESTAGIO DA TURBINA A VAPOR N6 DA ARCELORMITTAL TUBARÃO*
}

\author{
Eduardo Luiz Sales Marinho ${ }^{1}$ \\ Rogélio Dias $^{2}$ \\ João Victor de Carvalho Bomfim ${ }^{3}$
}

\section{Resumo}

A Central Termelétrica SOL, ou CTEs 5 e 6, foi construída com o objetivo de fazer o aproveitamento da energia do gás oriundo do processo de coqueificação do carvão mineral realizado em fornos específicos para este fim, em um processo denominado "Coqueria Heat Recovery". Nesta unidade operacional de responsabilidade da área de energia da ArcelorMittal Tubarão, seguindo o plano plurianual de manutenção nas turbinas Fuji, foi realizado intervenção de manutenção nos dois turbo gerados CTE's 05 e 06 no ano de 2015. Durante as atividade de inspeção de manutenção foi detectado que existiam trincas nos pés das palhetas encaixadas no rotor da turbina $n^{\circ} 6$. A partir desta constatação, 0 equipamento ficou sem confiabilidade para operação continua, precisando de substituição das palhetas danificadas. $O$ trabalho proposto apresenta as alternativas possíveis até a solução definitiva que foi a substituição das palhetas defeituosas por novos spare parts. As possibilidades descritas a seguir, serão o objeto de tomada de decisão até a normalização do equipamento: i) Montar TG e operar com palhetas do ultimo estágio trincadas até a chegada do sobressalentes para troca; ii) Deixar equipamento parado aguardando chegada dos sobressalentes para troca e conclusão da montagem e testes; iii) Remover por usinagem de campo, o ultimo estágio de palhetas do rotor montagem e operação do TG6 até a chegada do sobressalentes vindos da Fuji Japão.

Palavras-chave: Turbina; Palhetas; Trincas; Usinagem de campo.

\section{FILED MACHINING BLADES LAST STAGE OF STEAM TURBINE NUMBER SIX} ARCELORMITTAL TUBARÃO

\section{Abstract}

Central Thermoelectric Sun or power plant number five and six, was constructed in order to make use of the energy of the gas from the coking process of coal carried out in specific ovens for this purpose, in a process called "Coke Heat Recovery". The power plant operation is responsibility for ArcelorMittal Tubarão energy area. Following the maintenance plan Fuji turbine, the work was carried out in two turbines in 2015. During maintenance inspection activity was detected there were cracks in the blades of last stage of turbine number six. The equipment was not reliable for continuous operation, requiring replacement of damaged blades. The proposed work shows the possible alternatives until final solution to replacement for new spare parts. The possibilities below: i) Assembly turbine and operate with the last stage vanes cracked until the arrival spare parts to change; ii) Leave standing equipment waiting for arrival of parts for exchange and completion of the assembly and testing; iii) Remove by field machining, the last rotor blade and turbine operation until the arrival spare parts from Fuji Japan.

Keywords: Turbine; Blades; Cracks; Field machining.

1 Graduação em Engenharia mecânica, na função de especialista de confiabilidade de equipamentos, área / departamento IGE / IDG, Empresa: ArcelorMittal Tubarão, Serra ES Brasil.

2 Graduação em Engenharia mecânica, na função de especialista de manutenção mecânica da área de energia, área / departamento IGE / IDG, Empresa: ArcelorMittal Tubarão, Serra ES Brasil.

3 Graduação em Engenharia mecânica, na função de especialista de manutenção mecânica da área de energia, área / departamento IGM / IDG, Empresa: ArcelorMittal Tubarão, Serra ES Brasil. 


\section{INTRODUÇÃO}

A Central Termelétrica SOL, ou CTEs 5 e 6, foi construída com o objetivo de fazer o aproveitamento da energia do gás oriundo do processo de coqueificação do carvão mineral realizado em fornos específicos para este fim, em um processo denominado "Coqueria Heat Recovery". O gás, chamado de Flue Gas, é um sub-produto natural do processo de Coqueificação neste sistema "Heat Recovery"

O Flue Gas é basicamente um gás sem poder de combustão, com resíduos de SOx, NOx e material particulado, dotado de grande poder energético, ou entalpia, na forma de calor (altas temperaturas).

O total dos ativos (Coqueria e Termelétrica) é propriedade da SOL Coqueria Tubarão S.A., empresa formada pela ArcelorMittal Tubarão e a empresa Norte americana SunCoke; a Central Termelétrica é operada pela ArcelorMittal Tubarão, que arrendou da SOL os ativos da unidade.

A capacidade de geração das CTE's 05 e 06 é de 196,6 WV/h. As unidades partiram em 05/2007 e operam desde então seguindo as recomendações de operação e manutenção do fabricante do equipamento a empresa Japonesa Fuji.

Seguindo o plano plurianual de manutenção nas turbinas Fuji, foi realizado intervenção de manutenção nos dois turbo geradores das CTE's 05 e 06 no ano de 2015. Durante as atividade de inspeção de manutenção foi encontrado trincas nos pés das palhetas do rotor da turbina $n^{\circ} 6$. A partir desta constatação, o equipamento ficou sem confiabilidade para operação continua, precisando de substituição das palhetas danificadas. $O$ trabalho realizado pela AMT proporcionou um funcionamento continuo do TG ${ }^{\circ} 6$ até a chegada do sobressalentes para troca , com a confiabilidade e segurança requerida pela operação.

As opções para tomada de decisão estão apresentadas a seguir:

1) Montar TG e operar com palhetas do ultimo estágio trincadas até a chegada do sobressalentes para troca;

2) Deixar equipamento parado aguardando chegada dos sobressalentes para troca e conclusão da montagem e testes;

3) Remover por usinagem de campo, o ultimo estágio de palhetas do rotor, montagem e operação do TG $n^{\circ} 6$ até a chegada do sobressalentes vindo da Fuji Japão.

\section{MATERIAIS E MÉTODOS}

O fluxograma da planta da coqueria Heat recoverry de uma forma macro, recebe o calor dos gases de combustão proveniente das baterias de Coque, sendo utilizado como fonte geradora de calor para geração de vapor nas oito caldeiras instaladas na planta. O vapor produzido é enviado as CTE's 05 e 06 da ArcelorMittal Tubarão e convertido em energia elétrica em seus dois turbo geradores. 


\section{Planta Coqueria Heat Recovery - Fluxograma Macro $\underset{\text { Arcelormitral }}{\text { in }}$}
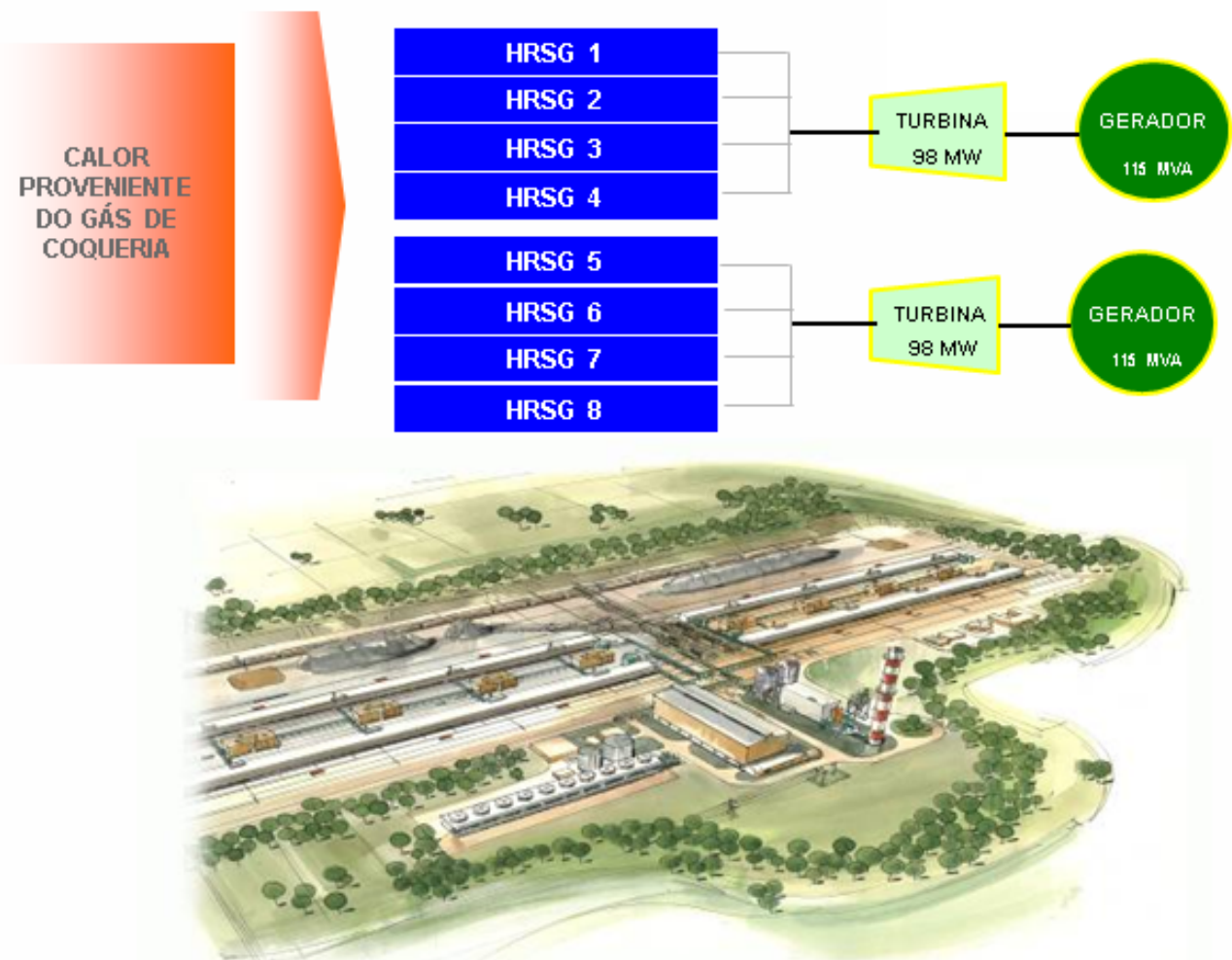

Fonte: ArcelorMittal Tubarão

\section{TURBINA}

Turbina é uma máquina rotativa que transforma a energia potencial (ou de pressão) do vapor em energia mecânica por intermédio da energia cinética, oriunda da expansão do vapor nas palhetas do rotor da Turbina. Basicamente as turbinas a vapor são constituídas de duas partes principais: rotor e estator. O estator é o elemento fixo destinado a transformar a energia potencial (ou térmica) do vapor em energia cinética. $O$ rotor é o elemento móvel destinado a transformar a energia cinética em mecânica.

O Gerador é acoplado ao eixo da Turbina e a energia mecânica é transformada em energia elétrica.

\section{TURBOGERADORES CTE SOL CARACTERÍSTICA}

\section{TURBINA}

Tipo Condensador Multi Estágios com Carcaça Simples

Potência Nominal:

Pressão de Entrada:

Temperatura de Entrada:

$98000 \mathrm{KW}$

№ 1 Pressão de Extração de Vapor:

$100.0 \mathrm{bar}$

№ 2 pressão de Extração de Vapor:

$532{ }^{\circ} \mathrm{C}$

Pressão de Exaustão:

6,35 bar

Rotação:

1,22 bar

0,107 bar

$3600 \mathrm{rpm}$ 
GERADOR

Tipo GTLRI 544/59-2

Saída:

$15600 \mathrm{KVA}$

Tensão:

$13800 \mathrm{~V}$

Corrente:

$4836 \mathrm{~A}$

$\mathrm{N}^{\circ}$ de fases:

3

Fator de Potência: 0,85

No de Polos: $\quad 2$

Frequência: $\quad 60 \mathrm{~Hz}$

\section{CARACTERIZAÇÃO DO PROBLEMA}

Durante a inspeção do rotor da turbina $n^{\circ} 6$, em 27/07/15 foram encontradas trincas em 29 palhetas do último estágio conforme mostrado abaixo: (total 46 palhetas)

G. $\mathbf{S}$

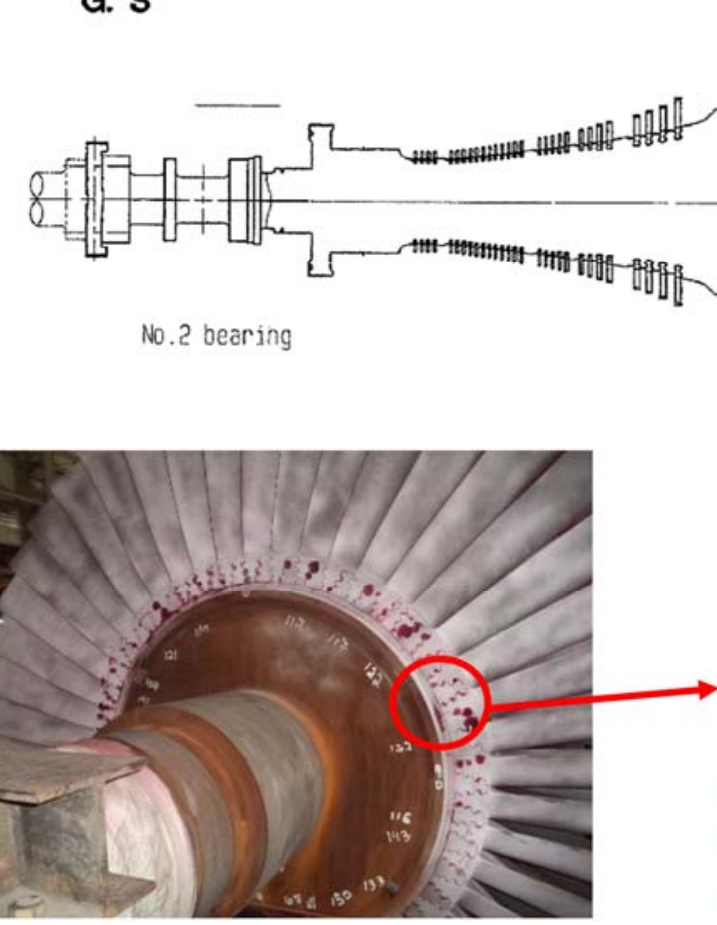

C. $S$

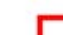

Fonte: ArcelorMittal Tubarão

\section{HISTÓRICO DE MANUTENÇÃO DO EQUIPAMENTO}

A recomendação de manutenção do fabricante pede uma abertura para inspeção de componentes a cada 04 anos e inspeção de integridade de carcaça, rotor, válvulas entre 10 e 12 anos, aplicando ensaios não destrutivos. $O$ histórico de manutenção de o equipamento a seguir mostra que as manutenções de inspeção da turbina que estavam sendo respeitadas conforme orientação do fabricante. 


\section{Inspeções realizadas na turbina 06}

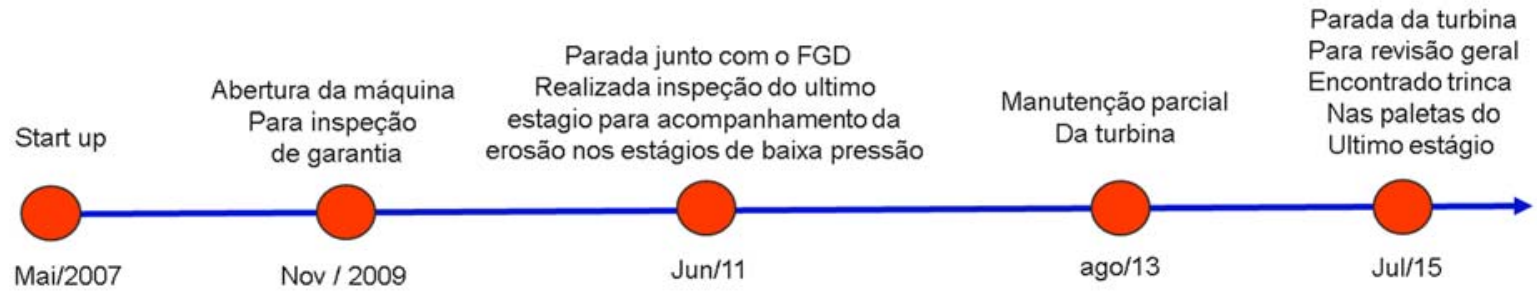

Fonte: ArcelorMittal Tubarão

\section{OPÇÕES DE MANUTENÇÃO ATÉ A TROCA DAS PALHETAS DO ROTOR}

1 ) Montar TG e operar com palhetas do ultimo estágio trincadas até a chegada do sobressalentes para troca:

Não aceito pois colocaria em risco a segurança das pessoas assim como difícil mensurar qual a perda material causada ao ativo caso ocorra uma quebra de palhetas do rotor em operação.

2 ) Deixar equipamento parado aguardando chegada dos sobressalentes para troca e conclusão da montagem e testes 27 de agosto até 13 de setembro:

Não aceito pois iria aumentar a perda de geração de energia em mais 16 dias até que o sobressalente chegue do Japão e possa ser montado.

3 ) Remover Por usinagem de campo, o ultimo estágio de palhetas do rotor montagem e operação do TG6 até a chegada do sobressalentes vindo da Fuji Japão.

Aceito por dois motivos:

$1^{\circ}$ Dezesseis dias de geração até a chegada do sobressalentes ;

$2^{\circ}$ Desmontagem parcial da capota da turbina , reduzindo o tempo de colocação da unidade em operação após troca das palhetas do ultimo estágio;

\section{USINAGEM DE CAMPO}

Ponto de atenção do serviço:

O corte manual poderia danificar eixo e ou palhetas do estágio anterior. Instalado as proteções físicas conforme ilustrado a seguir:

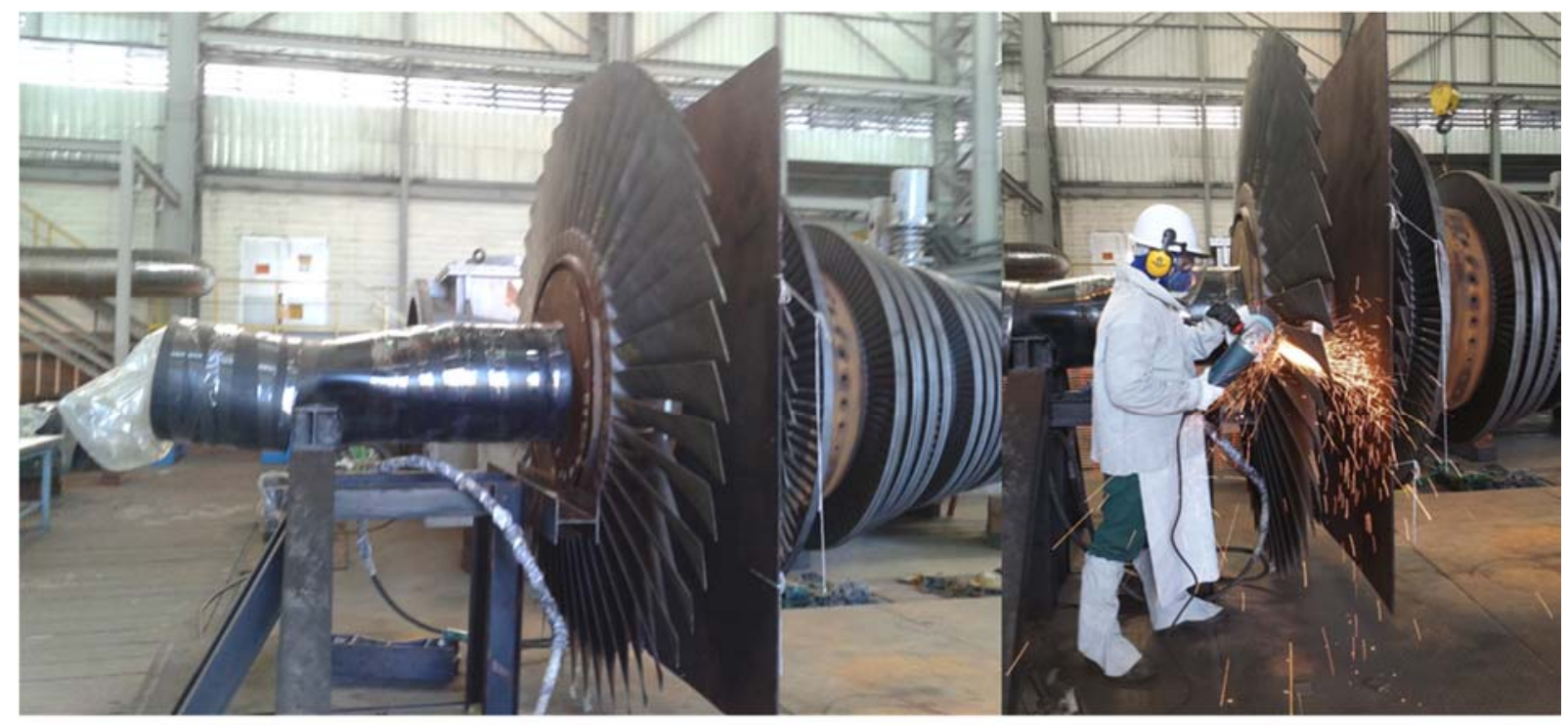

Preparação para corte de palhetas, proteção do eixo e palhetas do estágio anterior
Corte manual das palhetas do rotor ( 46 palhetas)

Fonte: ArcelorMittal Tubarão 
Não conseguir rodar o eixo garantindo sua posição geométrica concentrica no apoio de ambos os mancais no decorrer da usinagem. Fabricado e instalado lunetas de apoio do rotor, verificação de nivelamento, excentricidade e instalação de apoio axial para garantia não haver deslocamento do eixo durante usinagem.

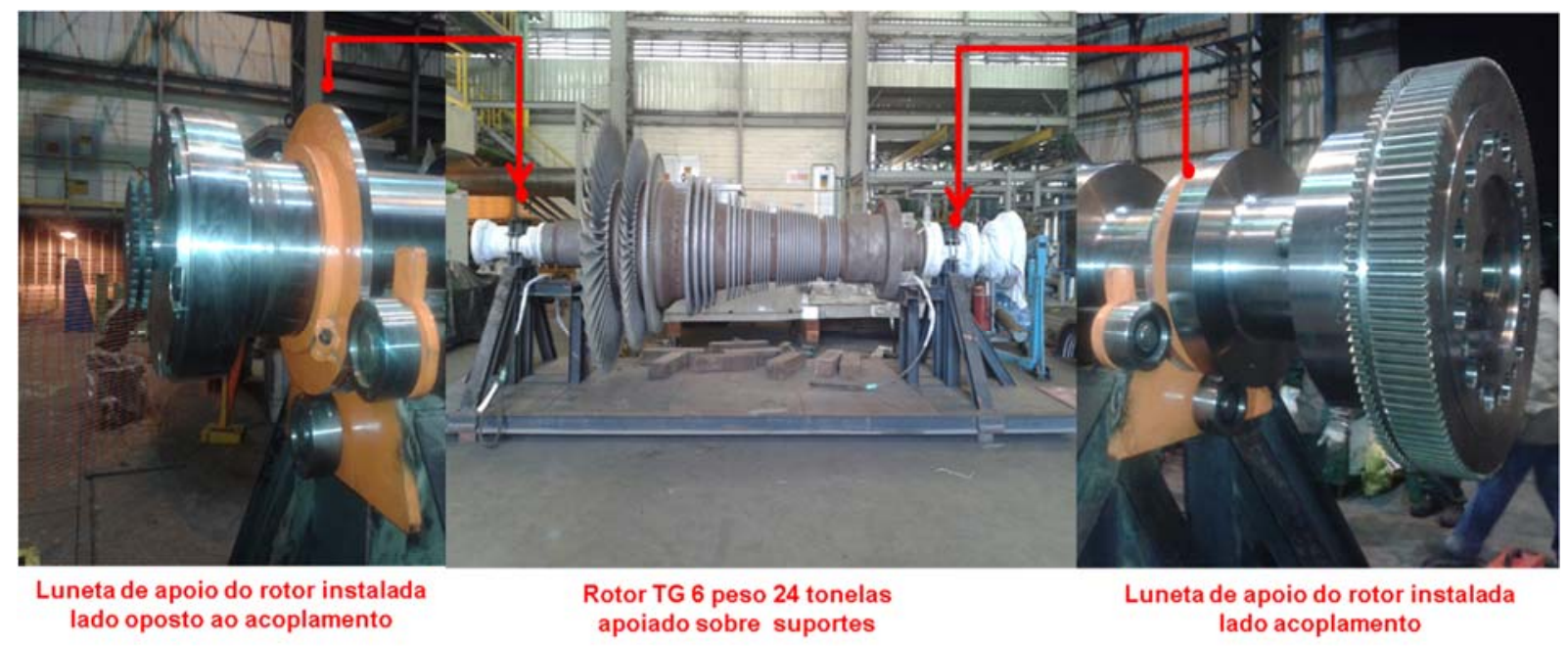

Fonte: ArcelorMittal Tubarão

Risco de variação dimensional no corte individual de palhetas, que iria ocasionar um desbalanço de massa no eixo e consequente vibração em operação em função do aumento da força de excitação do eixo $\mathbf{F}=\mathbf{m} \boldsymbol{\omega}^{2} \mathbf{r}$.

Instalado equipamento de usinagem de campo para fresagem de topo das palhetas. Definido a altura de corte da palheta em conjunto com o fabricante do equipamento. Instalado batente mecânico para garantir dimensional de usinagem.

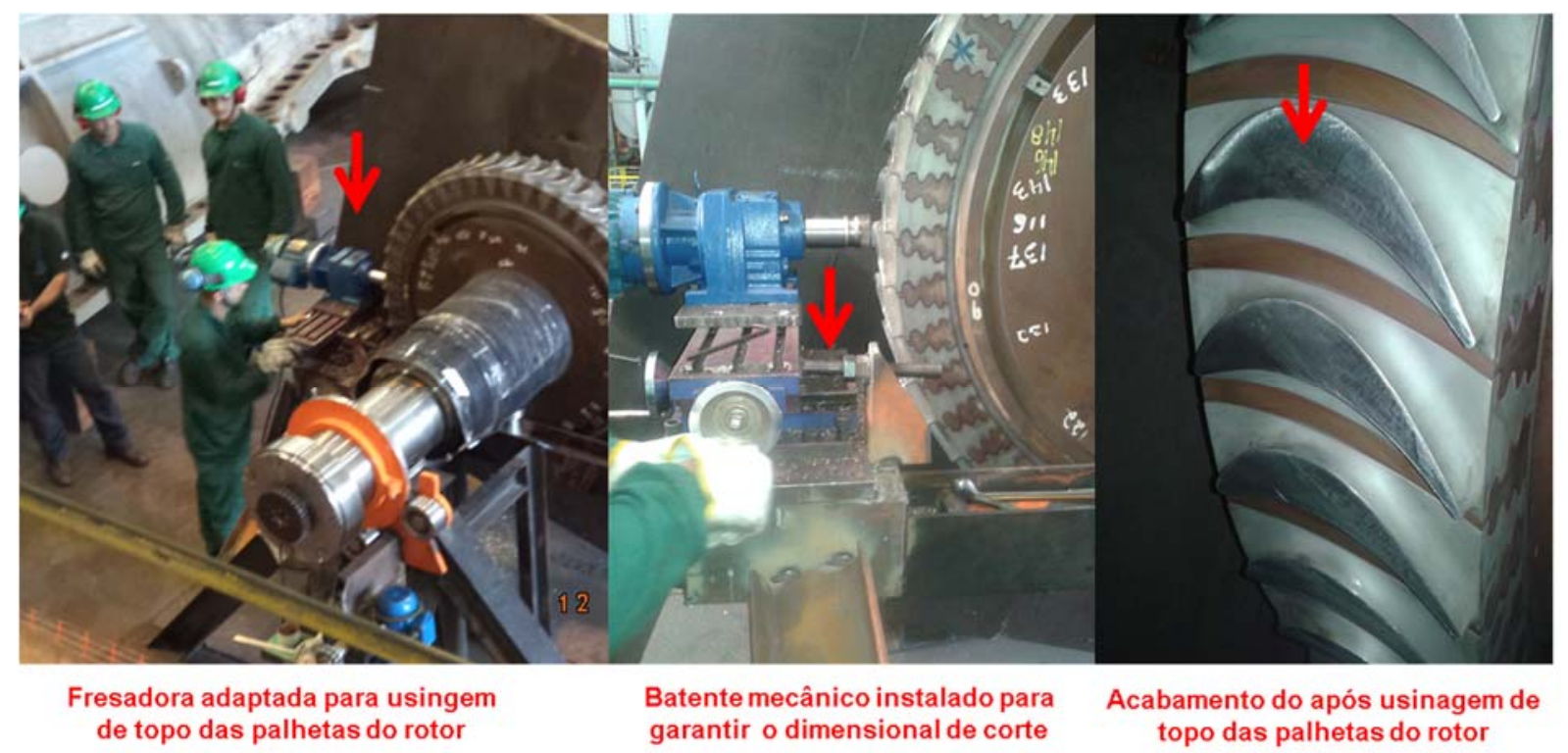

Fonte: ArcelorMittal Tubarão

\section{RESULTADOS E DISCUSSÃO}

Após montagem e partida da unidade os níveis de vibração aumentaram, mas ficaram dentro dos níveis aceitáveis por norma, até a troca definitiva das palhetas. 


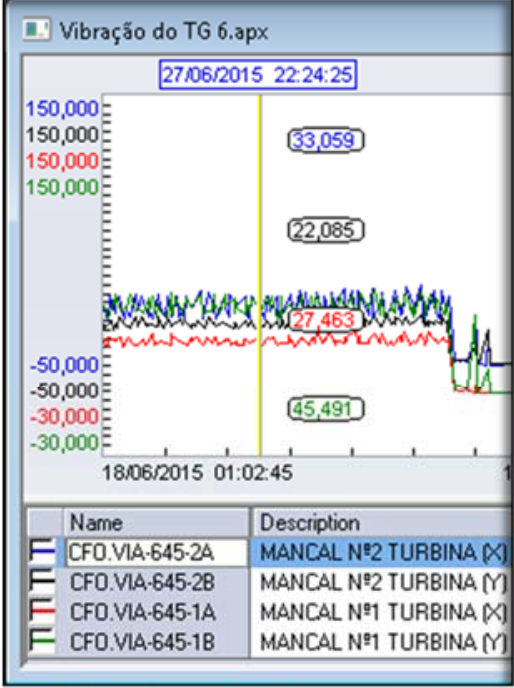

Vibração antes da manutenção ( Max $41 \mu$ )

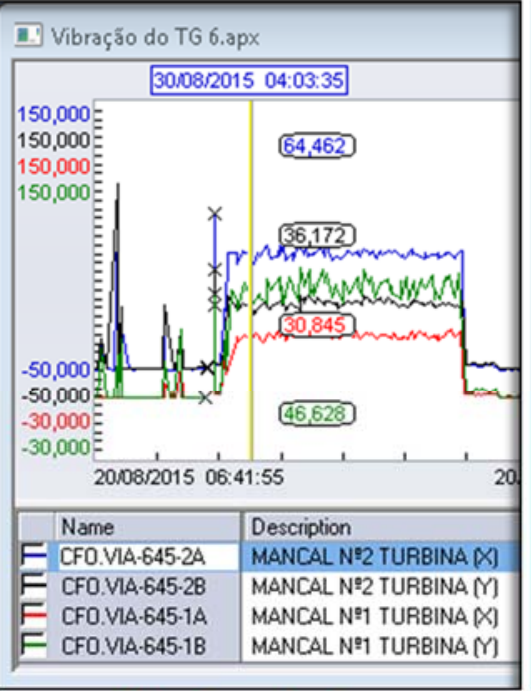

Vibração após usingem $(\operatorname{Max} 64 \mu)$

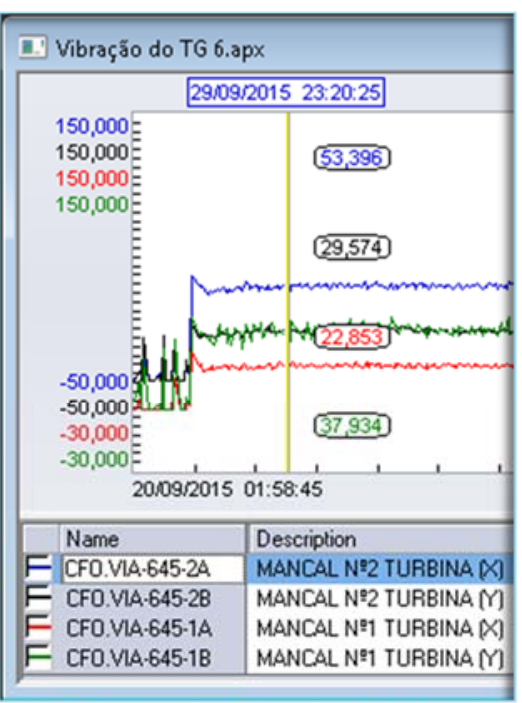

Vibração após trocar palhetas $(\operatorname{Max} 64 \mu)$

Fonte: ArcelorMittal Tubarão

A unidade operou de 28 de agosto de 2015 durante 16 dias com uma geração média de 65.47 MW, contabilizando um ganho de $\mathrm{R} \$ 3.570 .000,00$.

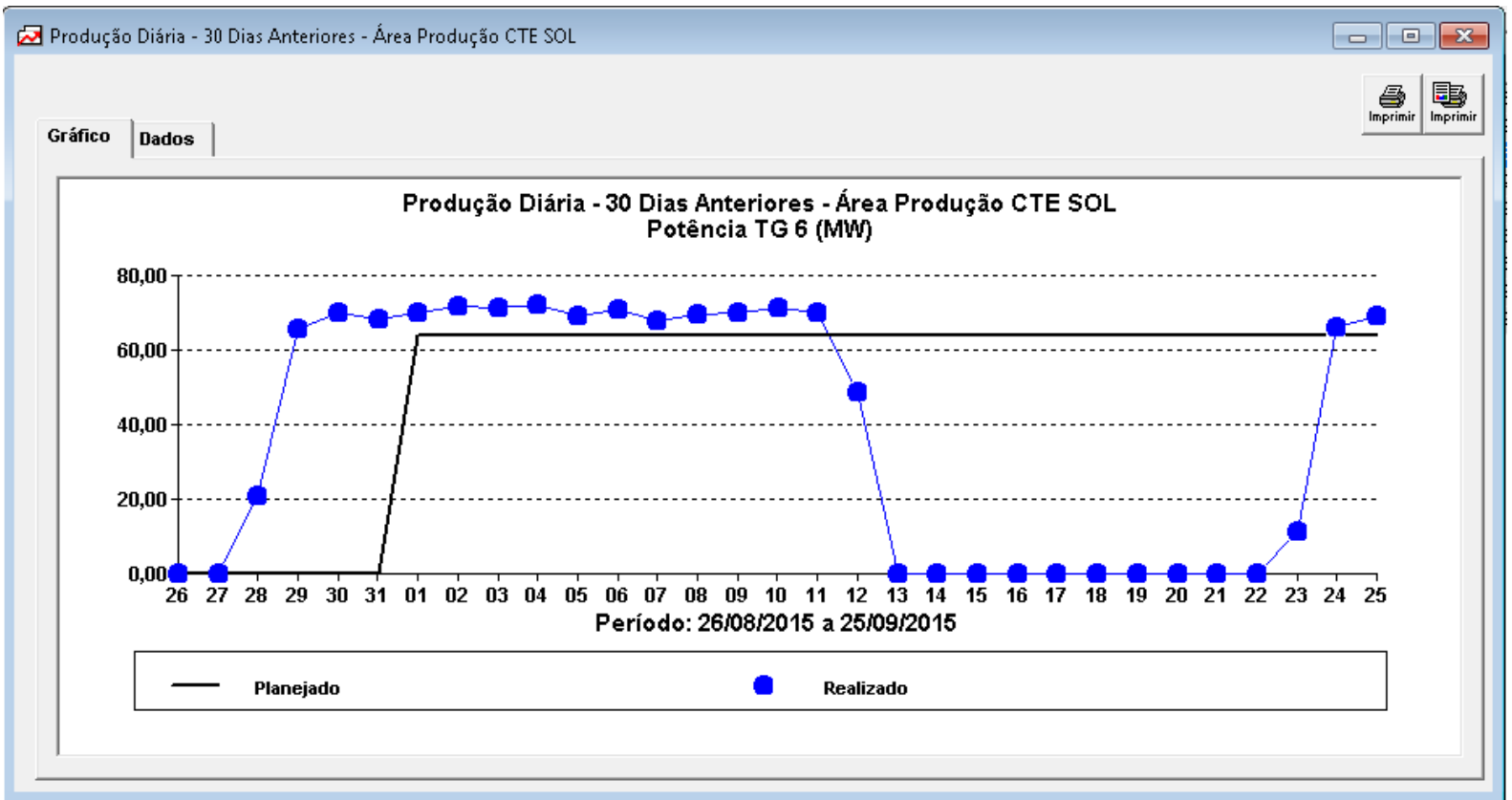

Fonte: ArcelorMittal Tubarão

\section{CONCLUSÃO}

O trabalho apresentado atingiu os dois objetivos desejados. Operou dentro de limites aceitáveis de vibração e gerou um resultado de $R \$ 3.570 .000,00$,período de 16 dias. Destaque para identificação e bloqueio dos riscos na operação de usinagem de campo mencionados acima.

Destaque para a visualização de uma solução de um problema de manutenção, mas com a preocupação de não estar causando ou imputando outro problema a partir da solução adotada. 


\section{Agradecimentos}

A gerencia de planejamento e inspeção da área de energia, Eng. Adilson Ferrarini, a gerencia de produção da área de energia, Eng. Luiz Enrique Sturião, a gerencia de manutenção da área de energia Eng. Tarley Secchin e o Eng. Fabricio de Assis gerente da planta de energia da ArcelorMittal Tubarão, que apoiaram tecnicamente e assumiram juntos com os engenheiros de manutenção de área, a iniciativa de reduzir o tempo de maquina parada, minimizando os impactos com a perda da geração de energia.

\section{REFERÊNCIAS}

1 Fabricante do turbo gerador Fuji Japão

2 ISO 1940-1 -Mechanical vibration -Balance quality requirements for rotors in a constant (rigid) state 